\title{
Teor e composição do óleo essencial de quatro acessos e duas cultivares de manjericão (Ocimum basilicum L.)
}

\author{
VELOSO, R.A. ${ }^{*}$; CASTRO, H.G. 1; BARBOSA, L.C.A; CARDOSO, D.P. 1; CHAGAS JÚNIOR, A.F. 1; SCHEIDT, \\ G.N. ${ }^{\text {. }}$ \\ Universidade Federal do Tocantins, Campus de Gurupi, Rua Badejós, Chácaras 69 a 72, Zona Rural, CEP \\ 77402-970, Caixa Postal 66, Gurupi, TO. 'Universidade Federal de Viçosa, Departamento de Química, Campus \\ de Viçosa, Centro, CEP 36571-000, Viçosa-MG; Universidade Federal de Minas Gerais, Departamento de \\ Química, (ICEx), Av. Pres. Antônio Carlos, 6627, Campus Pampulha, CEP 31270-901, Belo Horizonte, MG, \\ Brasil. *roniceuft@hotmail.com
}

\begin{abstract}
RESUMO: O objetivo desse estudo foi avaliar o teor e a composição química dos óleos essenciais de quatro acessos silvestres de manjericão (Ocimum basilicum L.), provenientes de diferentes regiões geográficas, e duas cultivares comerciais cultivados sob as mesmas condições edafoclimáticas. As sementes dos acessos foram coletadas nos município de Gurupi-TO (GUR) e de Monte Alegre-GO (MAL, MVE e FPS) e as sementes da cultivar comercial manjericão roxo (MR) foram adquiridas no comércio de Gurupi e da cultivar Maria Bonita (MB) foram fornecidas pelo Horto de Plantas Medicinais da Universidade Federal de Lavras. O plantio foi realizado em vasos com capacidade de dez litros utilizando-se como substrato: solo de barranco e esterco bovino na proporção 2:1. O óleo essencial foi obtido por hidrodestilação em aparelho tipo Clevenger e a identificação e a porcentagem relativa dos compostos do óleo essencial foi realizada por Cromatografia à Gás acoplada a Detector de Espectrometria de Massas. Foram identificados trinta e nove substâncias nos óleos essenciais obtidos, abrangendo monoterpenos, sesquiterpenos e fenilpropanoides. Nas cultivares MB e MR o composto majoritário foi o linalol, enquanto nos acessos silvestres (MAL, GUR, FPS e MVE) o composto em maior quantidade foi o $(E)$-cinamato de metila. $O$ maior teor de óleo essencial foi obtido na cultivar Maria Bonita $(3,77 \%)$.
\end{abstract}

Palavras-chave: Ocimum basilicum, óleo essencial, linalol, constituição química, recursos genéticos vegetais

ABSTRACT: Essential oil composition and content of different accessions and cultivars of basil (Ocimum basilicum L.). The objective of this study was to evaluate the essential oil chemical composition and content of four wild accessions of basil (Ocimum basilicum L.), from different geographical regions, and two commercial cultivars grown under the same edaphoclimatic conditions. The seeds of the accessions were collected in the municipality of Gurupi, state of Tocantins (GUR), and Monte Alegre, state ofGoiás (MAL, MVE and FPS), and the seeds of the commercial cultivar of purple basil (MR) were acquired in the Gurupi market and the seeds of the commercial cultivar Maria Bonita (MB) were provided by the Garden of Medicinal Plants of the Federal University of Lavras. The seeds were planted in pots with a capacity of ten liters using as substrate soil from earth works and cattle manure in the proportion of 2:1. The essential oil was obtained by hydrodistillation using a Clevenger-type apparatus, and the identification and the relative percentage of the compounds of the essential oil were performed by Gas Chromatography coupled to Mass Spectrometry Detector. Thirty-nine compounds were identified in the essential oils obtained, which were divided into monoterpenes, sesquiterpenes and phenylpropanoids. In the cultivars MB and MR, the major compound was linalool, and in the wild accessions (MAL, GUR, FPS and MVE) it was the (E)-methyl cinnamate. The highest content of essential oil was obtained from the cultivar Maria Bonita (3.77\%).

Keywords: Ocimum basilicum, essential oil, Linalool, chemical composition, plant genetic resources 


\section{INTRODUÇÃO}

A variação genética em populações naturais de plantas e animais é a base da sua resistência perante às pressões do ambiente, sendo a existência de diferenças genéticas que permite a seleção natural. As plantas que ocorrem em condições edafoclimáticas distintas variam também quanto à sua constituição genética e atividade fisiológica, condicionadas pelo processo de seleção natural; embora pertencendo à mesma espécie, podem responder de modo muito diferente quando cultivadas em diferentes ambientes. A diversidade genética envolve, portanto, o metabolismo dos organismos e seus produtos (Castro et al., 2004b; Pires \& Gripp, 1988; Ottai et al., 2012).

A diferença na composição química encontrada por diferentes autores pode estar relacionada com fatores ambientais, genéticos e fenológicos (Castro et al., 2004a; Martins et al., 2006). Dessa forma, para se entender melhor as variações encontradas na composição química das plantas, todas as espécies previamente investigadas deveriam ser cultivadas e analisadas em condições iguais e controladas.

Entre os produtos do metabolismo secundário das plantas encontram-se os óleos essenciais que são misturas complexas que podem conter mais de uma centena de compostos orgânicos. Seus constituintes químicos podem pertencer às mais diversas classes de compostos, porém os terpenos são as classes mais encontradas. Os fenilpropenos, metabólitos derivados da rota do ácido chiquímico, também são frequentemente encontrados como constituinte dos óleos essenciais (Gonçalves et al., 2003; Castro et al., 2004a; Castro et al., 2010; Loayza et al., 1995).

Estudos em quimiotaxonomia consistem na investigação de compostos químicos que ocorrem em plantas, com o objetivo de evidenciar ou não a relação entre elas, devendo a ênfase ser dada aos compostos predominantes da espécie. A padronização das épocas de colheita, da parte colhida e do cultivo sob as mesmas condições ambientais auxiliam na identificação de variedades que apresentam diferenças na sua composição química (Castro et al., 2004b; Klein et al., 2009; Silva et al., 2010). Essa padronização é importante uma vez que condições de cultivo e a época de colheita podem interferir na composição dos óleos essenciais produzidos (Barbosa et al., 2007; Demuner et al., 2011; Martins et al., 2006).

O manjericão (Ocimum basilicum L.), pertencente à família Lamiaceae, é uma planta anual originária do Sudoeste Asiático e da África Central, utilizada como planta medicinal, aromática, apresentando substâncias de interesse para as indústrias alimentícia, farmacêutica, cosmética e também para a medicina tradicional, principalmente devido ao teor e composição química do seu óleo essencial (Silva et al., 2005; Hanif, et al., 2011; Rosado et al., 2011). Trabalhos realizados com a composição de óleos essenciais em variedades de manjericão identificaram como constituintes majoritários metil chavicol, linalol, geranial e estragol (Sajjadi, 2006; Ottai et al., 2012).

A composição dos óleos essenciais extraídos das folhas e dos ápices com inflorescência do manjericão varia de acordo com a constituição genética da planta e a localização geográfica. Em função de suas propriedades terapêuticas, o manjericão é amplamente utilizado na medicina popular como antiespasmódico, antitérmico, auxiliando também na digestão e no combate de infecções bacterianas e parasitárias intestinais (Martins et al., 2010).

A caracterização da constituição do óleo essencial de $O$. basilicum permite gerar informações para a obtenção de variedades mais adequadas ao uso terapêutico e com maior teor de óleo essencial. Essas variedades podem ser selecionadas para serem incluídas no programa de melhoramento genético de $O$. basilicum para obtenção de variedades adaptadas ao cultivo em determinadas regiões. As variedades melhoradas constituem alternativas econômicas para os agricultores, tendo em vista o estabelecimento de um sistema de produção sustentável diversificado com a integração do cultivo de espécies medicinais com outras culturas de interesse.

Assim, o objetivo desse estudo foi determinar o teor e a composição química do óleo essencial de quatro acessos silvestres de manjericão, provenientes de diferentes regiões geográficas, e duas cultivares comerciais cultivados sob as mesmas condições edafoclimáticas, visando identificar os acessos ou as cultivares com maior teor de óleo essencial e dos constituintes do seu óleo essencial.

\section{MATERIAL E MÉTODOS}

Foram utilizadas quatro acessos silvestres e duas cultivares comerciais cultivados em casa de vegetação da Universidade Federal do Tocantins, campus de Gurupi-TO. O plantio foi realizado em vasos com capacidade de dez litros, utilizando-se como substrato solo de barranco e esterco bovino na proporção 2:1. Aos 102 dias após o plantio foi realizado o corte das plantas de manjericão rente ao solo e a parte aérea foi colocada para desidrata àa temperatura ambiente para, posteriormente, ser realizada a extração do óleo essencial.

A composição química do substrato utilizado

Rev. Bras. Pl. Med., Campinas, v.16, n.2, supl. I, p.364-371, 2014. 
no experimento foi: $\mathrm{pH}\left(\mathrm{H}_{2} \mathrm{O}\right)=7,6 ; \mathrm{H}^{+}+\mathrm{Al}^{+++}=$ $0,99 \mathrm{cmol}_{\mathrm{c}} \mathrm{dm}^{-3} ; \mathrm{Ca}^{++}=2,73 \mathrm{cmol}_{\mathrm{c}} \mathrm{dm}^{-3} ; \mathrm{Mg}^{++}=2,14$ $\mathrm{cmol}_{\mathrm{c}} \mathrm{dm}^{-3} ; \mathrm{P}$ (extrator Melich 1) $=107,4 \mathrm{mg} \mathrm{dm}^{-3}$; $\mathrm{K}^{+}=798,4 \mathrm{mg} \mathrm{dm}^{-3}$; matéria orgânica $=66,64 \mathrm{~g} \mathrm{dm}^{-3}$; CTC $=6,92 \mathrm{cmol}_{c} \mathrm{dm}^{-3}$ e V\%= 87,48\%.

As sementes dos acessos silvestres foram coletadas nos municípios de Gurupi-TO (GUR) $\left(11^{\circ} 43^{\prime} 30^{\prime \prime}\right.$ sul e $49^{\circ} 04^{\prime} 34^{\prime \prime}$ oeste, com altitude de $287 \mathrm{~m}$ ) e de Monte Alegre-GO. No município de Monte Alegre foram coletados três acessos: o acesso MAL foi coletado no bairro Central $\left(13^{\circ} 15^{\prime} 24^{\prime \prime}\right.$ sul e $46^{\circ} 53^{\prime} 24^{\prime \prime}$ oeste, com altitude de $543 \mathrm{~m}$ ), o acesso MVE foi coletado no bairro Montes Verdes $\left(13^{\circ} 15^{\prime} 36^{\prime \prime}\right.$ sul e $46^{\circ} 52^{\prime} 53^{\prime \prime}$ oeste, com altitude de $548 \mathrm{~m}$ ) e o acesso FPS foi coletado na Fazenda Ponta da Serra $\left(13^{\circ} 13^{\prime} 05^{\prime \prime}\right.$ sul e $46^{\circ} 48^{\prime} 24^{\prime \prime}$ oeste, com altitude de $577 \mathrm{~m}$ ). Para a cultivar comercial de manjericão roxo (MR) as sementes foram adquiridas no comércio local de Gurupi-TO e as sementes da cultivar Maria Bonita (MB) foram fornecidas pelo Horto de Plantas Medicinais da Universidade Federal de Lavras.

As exsicatas dos acessos e das cultivares foram confeccionadas e depositadas no herbário da Universidade Federal de Lavras com os números: MAL - 26.599; GUR - 26.603; FPS - 26.602; MVE 26.601; MB - 26.920; e MR - 26.600.

O óleo essencial foi obtido por hidrodestilação a partir de amostras da parte aérea de plantas de manjericão desidratadas $(0,02 \mathrm{~kg})$ por um período de duas horas, com quatro repetições. As amostras foram colocadas em balão de fundo redondo contendo $1 \mathrm{~L}$ de água destilada, acoplado ao aparelho tipo Clevenger e este, a um condensador. O óleo essencial foi separado da fase aquosa, utilizando-se pentano $(4 \times 40 \mathrm{~mL})$. As frações orgânicas obtidas foram reunidas e secadas com sulfato de magnésio anidro, filtradas, e o solvente foi removido sob pressão reduzida em evaporador rotativo a $40^{\circ} \mathrm{C}$, em equipamento QUIMIS, modelo Q344B2 (Brasil, São Paulo), em seguida, o óleo essencial foi pesado e efetuada a sua quantificação (Veloso et al., 2012).

Os dados do teor de óleo essencial foram interpretados por meio de análise de variância e as médias foram comparadas pelo teste de Tukey, a $5 \%$ de probabilidade (Ribeiro Júnior \& Melo, 2009).

A análise qualitativa dos óleos essenciais foi realizada por cromatografia em fase gasosa acoplada a espectrometria de massas (CG/EM) em equipamento Shimadzu, modelo QP 2010 Plus, com tempo de análise de 70 minutos, em coluna capilar Equily-5 (30 m x 0,25 mm x 0,25 $\mu \mathrm{m})$. Foi utilizado hélio como gás de arraste com a velocidade de 1,8 $\mathrm{mL} \mathrm{min}^{-1}$; taxa de Split 1:20 com volume injetado de $1 \mu \mathrm{L}$. As temperaturas do injetor e do detector foram $220^{\circ} \mathrm{C}$ e $240^{\circ} \mathrm{C}$, respectivamente. A temperatura inicial do forno foi de $60^{\circ} \mathrm{C}$ por $2 \mathrm{~min}$, seguido de um incremento de $3^{\circ} \mathrm{C}$ por min até atingir $240^{\circ} \mathrm{C}$, sendo mantida constante por 15 minutos. Em todas as análises a energia do feixe de elétrons foi de $70 \mathrm{eV}$.

A identificação dos constituintes químicos foi feita por comparação dos seus espectros de massa com os espectros de massa disponíveis no banco de dados do equipamento (biblioteca Willey 7), com a literatura e pelo índice aritmético de retenção relativo $(I A)$ à série de alcanos $C_{9}-C_{22}$ (Adams, 2007). A porcentagem relativa desses constituintes foi realizada pela integração da área dos cromatogramas de íons totais obtidos a partir da cromatografia gasosa acoplada a espectrometria de massas (Rosado et al., 2011).

\section{RESULTADOS E DISCUSSÃO}

Os maiores valores de teordeo óleo essencial foram observados na cultivar Maria Bonita $(3,77 \%)$ e na Manjericão Roxo $(3,15 \%)$, enquao e o menor valor foi observado para o acesso GUR $(1,52 \%)$, conforme pode ser observado na Tabela 1. O teor de óleo essencial obtido da parte aérea das plantas da cultivar Maria Bonita permite a projeção da produção de $438,19 \mathrm{~kg} \mathrm{ha}^{-1}$ de óleo essencial.

Aanálise do óleo essencial por cromatografia gasosa acoplaa aà espectrometria de massas resultou na identificação de trinta e nove constituintes no óleo essencial de $O$. basilicum, divididos em monoterpenos, sesquiterpenos e fenilpropanoides. Destes, o maior número de compostos identificados foi observado na cultivar MB, apresentando na constituição de seu óleo essencial 26 constituintes (Tabela 1).

Estudos realizados com os genótipos de

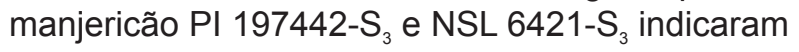
que o teor de óleo essencial de massa seca de folhas e inflorescências variou de 1,36 a 1,70\% e de 4,01 a $5,17 \%$, respectivamente, corroborando com os resultados obtidos neste trabalho (Luz et al., 2009). Posteriomente, estudos de Blank et al. (2010) avaliando o teor de óleo em quatro populações de manjericão (NSL 6421-S -Bulk $^{-}$

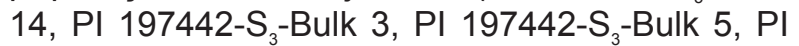

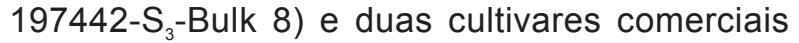
consideradas testemunha (Genovese e Osmin Purple) encontraram valores médios de $4,36 \%$ para as populações, demonstrando superioridade destas em relação às cultivares comerciais Genovese $(1,22 \%)$ e Osmin Purple $(1,31 \%)$. Nestes dois trabalhos os autores identificaram como constituinte majoritário o linalol.

Para fins de apresentação os compostos foram listados nos seguintes grupos: monoterpenos hidrocarbonetos; monoterpenos oxigenados; sesquiterpenos hidrocarbonetos; sesquiterpenos

Rev. Bras. PI. Med., Campinas, v.16, n.2, supl. I, p.364-371, 2014. 
TABELA 1. Teor do óleo essencial e porcentagem relativa (área \%), obtida por Cromatografia à Gás acoplada a Detector de Espectrometria de Massas, dos constituintes do óleo essencial da parte aérea de quatro acessos (MAL, GUR, FPS e MVE) e duas cultivares (MB e MR) de Ocimum basilicum.

\begin{tabular}{|c|c|c|c|c|c|c|c|c|c|}
\hline \multirow[b]{2}{*}{ Constituintes } & \multirow[b]{2}{*}{$\mathrm{NC}$} & \multirow[b]{2}{*}{ TR } & \multirow[b]{2}{*}{ IA } & \multicolumn{6}{|c|}{ Acessos/cultivares } \\
\hline & & & & MAL & GUR & FPS & MVE & MB & MR \\
\hline \multicolumn{10}{|l|}{ Monoterpenos } \\
\hline \multicolumn{10}{|l|}{ hidrocarbonetos } \\
\hline$\alpha-$ pineno & 1 & 5,72 & 931 & - & - & - & - & 0,22 & - \\
\hline Sabineno & 2 & 6,81 & 969 & - & - & - & - & 0,37 & - \\
\hline$\beta$-pineno & 3 & 6,93 & 973 & - & - & - & - & 0,77 & - \\
\hline$\beta$-m irceno & 4 & 7,28 & 988 & - & - & - & - & 0,43 & - \\
\hline Limoneno & 5 & 8,58 & 1025 & 0,09 & - & 0,23 & - & 0,30 & - \\
\hline$\beta$-ocimeno & 6 & 9,22 & 1041 & - & - & - & - & 0,48 & - \\
\hline Subtotal & & & & 0,09 & 0 & 0,23 & 0 & 2,57 & 0 \\
\hline \multicolumn{10}{|l|}{ Monoterpenos } \\
\hline \multicolumn{10}{|l|}{ oxigenados } \\
\hline 1,8-cineol & 7 & 8,69 & 1027 & - & - & - & - & 13,09 & 2,63 \\
\hline Hidrato de sabineno & 8 & 9,97 & 1062 & - & - & - & - & 0,20 & 0,18 \\
\hline Óxido de linalol & 9 & 10,17 & 1066 & - & - & - & - & 0,07 & 0,07 \\
\hline Fenchona & 10 & 10,81 & 1079 & - & - & - & - & - & 0,47 \\
\hline Linalol & 11 & 11,28 & 1094 & 0,22 & 0,28 & 0,12 & 4,43 & 36,32 & 47,76 \\
\hline Cânfora & 12 & 13,05 & 1142 & - & - & - & - & 0,89 & 1,13 \\
\hline Nerol & 13 & 17,65 & 1230 & - & - & - & - & 0,80 & 0,58 \\
\hline Subtotal & & & & 0,22 & 0,28 & 0,12 & 4,43 & 51,37 & 52,82 \\
\hline \multicolumn{10}{|l|}{ Sesquiterpenos } \\
\hline \multicolumn{10}{|l|}{ hidrocarbonetos } \\
\hline a-cubebeno & 14 & 22,81 & 1345 & 0,09 & - & - & - & - & - \\
\hline a-copaeno & 15 & 23,63 & 1376 & 0,09 & 0,01 & 0,04 & 0,11 & 0,04 & - \\
\hline$\beta$-elemeno & 16 & 24,40 & 1394 & - & - & - & - & - & 2,88 \\
\hline E-cariofileno & 17 & 24,61 & 1402 & 5,18 & 1,33 & 2,66 & 6,72 & 0,49 & 1,21 \\
\hline a-bergamoteno & 18 & 25,27 & 1412 & 5,29 & 1,13 & 2,65 & 7,66 & 5,09 & 0,96 \\
\hline a-guaieno & 19 & 25,38 & 1440 & - & - & - & - & - & 0,69 \\
\hline (E)- $\beta$-farneseno & 20 & 26,11 & 1458 & 0,23 & - & 0,11 & 0,33 & - & - \\
\hline Germacreno D & 21 & 27,13 & 1483 & 0,12 & - & 0,05 & 0,15 & 1,06 & 1,14 \\
\hline$(E, E)$-a-Farneseno & 22 & 27,67 & 1496 & 1,19 & 0,31 & 0,45 & 2,73 & - & - \\
\hline (E)-ß-Guaieno & 23 & 28,10 & 1504 & - & - & - & - & 1,15 & 1,14 \\
\hline$\beta$-bisaboleno & 24 & 28,22 & 1499 & 0,11 & - & - & 0,20 & - & - \\
\hline$\delta$-amorfeno & 25 & 28,44 & 1516 & - & - & - & - & 0,75 & - \\
\hline Y-cadineno & 26 & 28,83 & 1510 & 0,76 & 0,14 & 0,28 & 1,19 & - & - \\
\hline Subtotal & & & & 13,06 & 2,92 & 6,24 & 19,09 & 8,58 & 8,02 \\
\hline
\end{tabular}


TABELA 1. Teor do óleo essencial e porcentagem relativa (área \%), obtida por Cromatografia à Gás acoplada a Detector de Espectrometria de Massas, dos constituintes do óleo essencial da parte aérea de quatro acessos (MAL, GUR, FPS e MVE) e duas cultivares (MB e MR) de Ocimum basilicum.

continuação...

\begin{tabular}{|c|c|c|c|c|c|c|c|c|c|}
\hline \multicolumn{10}{|l|}{ Sesquiterpenos } \\
\hline \multicolumn{10}{|l|}{ oxigenados } \\
\hline Estragol & 27 & 15,26 & 1201 & - & - & - & 0,16 & 28,41 & - \\
\hline Elemol & 28 & 29,82 & 1543 & - & - & - & - & 0,19 & - \\
\hline Nerolidol & 29 & 30,34 & 1559 & - & - & - & 0,38 & 0,06 & 0,15 \\
\hline Espatulenol & 30 & 30,94 & 1573 & - & - & - & - & - & 0,49 \\
\hline Óxido de cariofileno & 31 & 31,15 & 1574 & 0,15 & 0,04 & - & 0,88 & 0,05 & 0,27 \\
\hline Veridiflorol & 32 & 32,00 & 1596 & - & - & - & 0,34 & - & - \\
\hline Eudesmol 10-epi-y & 33 & 32,99 & 1624 & - & - & - & - & 0,05 & - \\
\hline Epi-a-Cadinol & 34 & 33,33 & 1641 & - & - & - & - & 2,19 & 3,14 \\
\hline$\beta$-eudesmol & 35 & 33,68 & 1648 & - & - & - & - & 0,18 & 0,33 \\
\hline a-cadinol & 36 & 33,86 & 1652 & - & - & - & 0,26 & - & - \\
\hline Subtotal & & & & 0,15 & 0,04 & 0 & 2,02 & 31,13 & 4,38 \\
\hline \multicolumn{10}{|l|}{ Fenilpropanóide } \\
\hline (Z)-cinamato de metila & 37 & 19,89 & 1299 & 3,04 & 2,46 & 3,88 & 24,15 & - & 0,96 \\
\hline Eugenol & 38 & 22,05 & 1356 & - & - & - & - & - & 2,25 \\
\hline$(E)$-cinamato de metila & 39 & 23,11 & 1369 & 80,93 & 93,59 & 88,65 & 39,23 & 1,04 & 22,22 \\
\hline Subtotal & & & & 83,97 & 96,05 & 92,53 & 63,38 & 1,04 & 25,43 \\
\hline Total identificado & & & & 97,49 & 99,29 & 99,12 & 88,92 & 94,69 & 90,65 \\
\hline Teor de óleo* (\%) & & & & $2,10 \mathrm{c}$ & $1,52 \mathrm{~d}$ & $1,97 \mathrm{~cd}$ & $1,8 \mathrm{~cd}$ & 3,77 a & $3,15 b$ \\
\hline
\end{tabular}

$\mathrm{NC}=$ número dos compostos. IA = Índice aritmético de retenção calculado. * teor de óleo essencial expresso em base seca. Médias seguidas pela mesma letra na linha, na variável teor de óleo, não diferem entre si pelo teste de Tukey $(P>0,05)$.

oxigenados; e fenilpropanóides. Verificou-se que nas cultivares MB e MR foi obtida a maior porcentagem relativa de monoterpenos, $53,94 \%$ e $52,82 \%$, respectivamente. Nos acessos silvestres (MAL, GUR, FPS e MVE) foi obtida a maior porcentagem relativa de fenilpropanoides (Tabela 1 e Figura 1).

Foram identificados dois constituintes majoritários presentes nos óleos essenciais das amostras avaliadas:um monoterpeno (linalol), majoritário nas duas cultivares, eum fenilpropanoide $((E)$-cinamato de metila), majoritário nos acessos silvestres. Outros compostos obtidos em quantidades expressivas foram os sesquiterpenos $E$-cariofileno e a-bergamoteno para os acessos ML e MVE e para a cultivar MB, e o monoterpeno 1,8-cineol e o sesquiterpeno oxigenado estragol apenas para a cultivar MB (Tabela 1 e Figura 2).

A predominância do fenilpropanoide cinamato de metila nos acessos silvestres sugere o deslocamento dos metabólitos primários da rota do ácido mevalônico para a rota do ácido chiquímico; essa suposição é reforçada pela presença de derivados terpênicos como o linalol, o 1,8-cineol e o estragol, que são substâncias majoritárias no óleo das variedades MB e MR, enquanto nos acessos silvestres essas substâncias ocorrem como traços. Cinamato é o precursor da maioria dos metabólitos originados da rota do chiquimato e da lignina que confere resistência à parede celular da planta, atuando no controle dos estresses físico e químico. Os fenilpropanoides apresentam funções importantes na defesa química do vegetal contra pragas, predadores e patógenos (Castro et al., 2004a; Friedrich, 1976; Matern \& Grimmig, 1994).

Resultados semelhantes a este trabalho foram encontrados por outros autores que analisaram a constituição química do óleo essencial do manjericão e obtiveram como constituintes majoritários o cinamato de metila e o linalol (González-Zúñiga et al., 2011). Em outro trabalho que se avaliou o óleo essencial de doze variedades

Rev. Bras. PI. Med., Campinas, v.16, n.2, supl. I, p.364-371, 2014. 


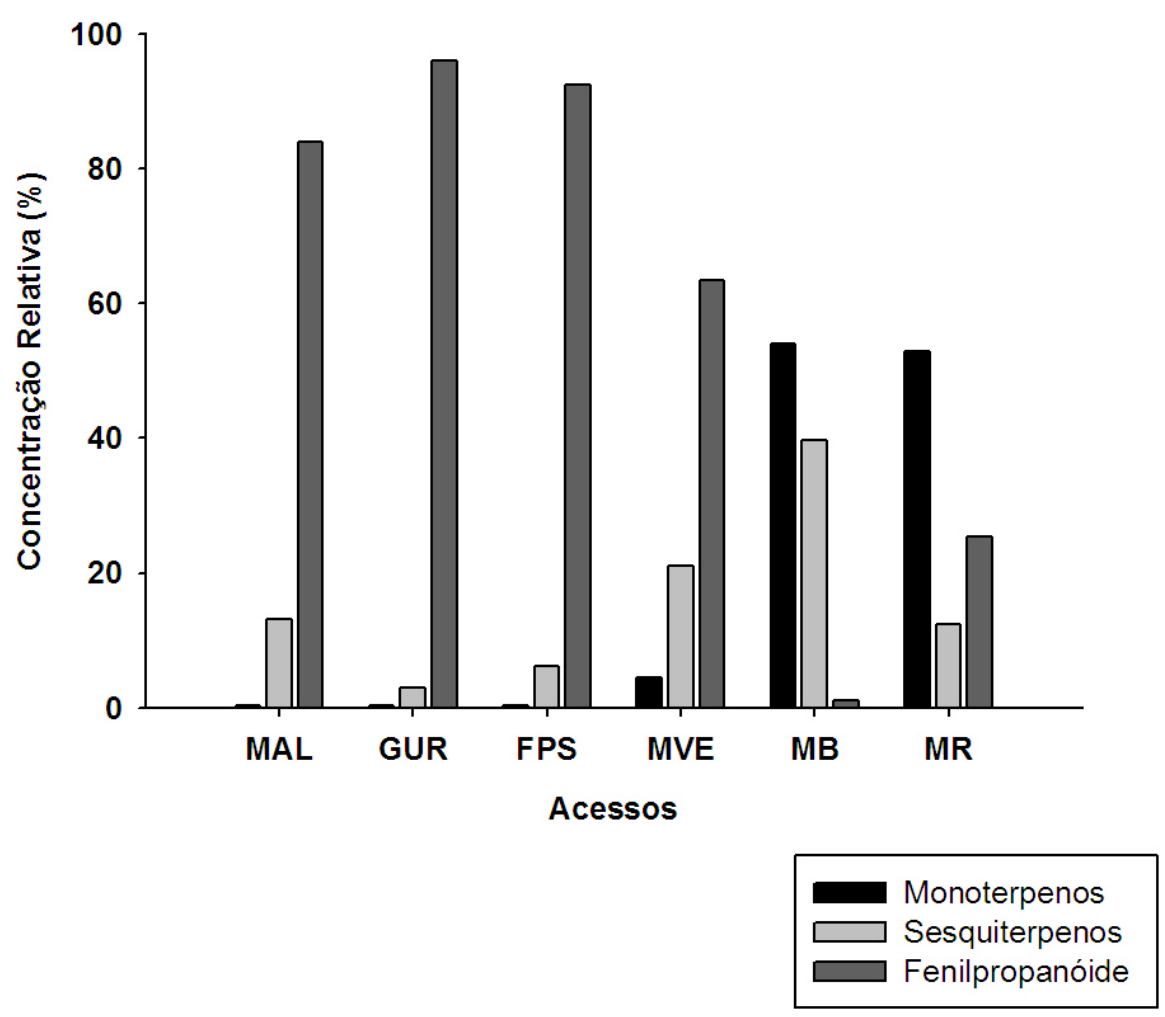

FIGURA 1. Porcentagem relativa de monoterpenos, sesquiterpenos e fenilpropanoide presentes no óleo essencial dos quatro acessos e dois cultivares de Ocimum basilicum L., cultivados em Gurupi-TO.

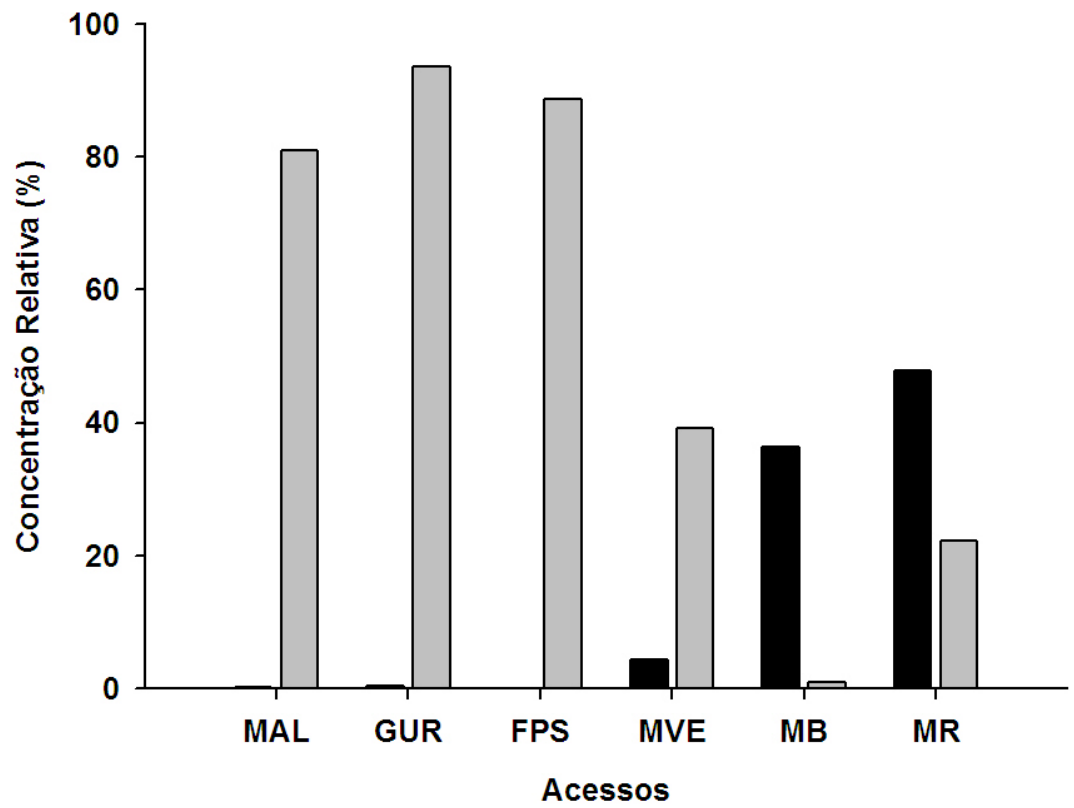

Linalol

(E) - Cinamato de metila

FIGURA 2. Porcentagem relativa (\%) dos constituintes majoritários do óleo essencial de quatro acessos e dois cultivares de Ocimum basilicum L.: linalol e $(E)$-cinamato de metila, cultivados em Gurupi-TO. 
de $O$. basilicum, foram identificados monoterpenos (45,6\%), sesquiterpenos (35,3\%) e fenilpropanoides $(7,4 \%)$ (Viña \& Murillo, 2003). Segundo os mesmos autores, os constituintes majoritários dos óleos essenciais foram o cinamato de metila e o linalol e Rosado et al. (2011) identificaram no óleo essencial da cultivar Maria Bonita o linalol como constituinte majoritário. Em todas as pesquisas citadas os resultados estão de acordo com aqueles obtido nesse estudo.

Altos teores de eugenol $(37-39 \%)$ e 1,8-cineol (18-21\%) foram obtidos no óleo de Ocimum selloi e, altos teores de metilchavicol (46$63 \%$ ) e linalol (24-33\%) foram encontrados no óleo essencial de Ocimum canum naturais da Bahia (Nascimento et al., 2011).

As cultivares comerciais Maria Bonita e Manjericão Roxo forneceram maiores teores de óleo essencial quando comparadas com os acessos silvestres avaliados. Em relação à composição química os acessos apresentaram como componente majoritário o fenilpropanoide $(E)$ cinamato de metila e as cultivares o monoterpeno oxigenado linalol. Esses resultados fornecem subsídios para a seleção de variedades adequadas de $O$. basilicum, tanto para o uso terapêutico devido a sua composição química, como para obtenção de variedades com alto teor de óleo essencial.

\section{AGRADECIMENTOS}

Ao Conselho Nacional de Desenvolvimento Científico e Tecnológico (CNPq), à Coordenação de Aperfeiçoamento de Pessoal de Nível Superior (Capes), pela concessão de bolsas e apoio financeiro e à Universidade Federal de Lavras pela disponibilização de sementes e realização das análises químicas dos óleos essenciais.

\section{REFERENCIA}

ADAMS, R.P. Identification of essential oil components by gas chromatography/mass spectroscopy, $4^{\text {th }}$ Edition. Illinois USA: Allured Publishing Corporation, Carol Stream, 2007, 804p.

BARBOSA, L.C.A.; DEMUNER, A.J.; DUMONT, A.C.; PAULA, V.F.; ISMAIL, F.M.D. Seasonal Variation in the Composition of Volatile Oils from Schinus terebinthifolius Raddi. Química Nova, v. 30, n. 8, p.1959 -1965, 2007.

BLANK, A.F.; SOUZA, E.M; PAULA, J.W.A.; ALVES, P.B.

Comportamento fenotípico e genotípico de populações de manjericão. Horticultura Brasileira. v.28, n.3, p.305310, 2010.

CASTRO, H.G.; FERREIRA, F.A.; SILVA, D.J.H.; MOSQUIM, P.R.; Contribuição ao estudo das plantas medicinais: metabólitos secundários. 2 ed. UFV: Viçosa, 2004a, 113p.

CASTRO, H.G.; OLIVEIRA, L.O.; BARBOSA, L.C.A.; FERREIRA, F.A.; SILVA, D.J.H.; MOSQUIM, P.R.;
NASCIMENTO, E.A. Teor e composição do óleo essencial de cinco acessos de mentrasto. Química Nova. v.27, n.1, p.55-57, 2004b.

CASTRO, H.G.; PERINI, V.B.M.; SANTOS, G.R.; LEAL, T.C.A.B. Avaliação do teor e composição do óleo essencial de Cymbopogon nardus (L.) em diferentes épocas de colheita. Revista Ciência Agronômica. v. 41, n. 2, p.308-314, 2010.

DEMUNER, A.J.; BARBOSA, L.C.A.; MAGALHÃES, C.G.; SILVA, C.J; MALTHA, C.R.A.; PINHEIRO, A.L. Seasonal variation in the chemical composition and antimicrobial activity of volatile oils of three species of Leptospermum (Myrtaceae) grown in Brazil. Molecules, v.16, n.2, p.1181-1191, 2011.

FRIEDRICH, H., Phenylpropanoid constituents of essential oil. Lloydia, v.39, n.1, p.1-7, 1976.

GONÇALVES, L.A.; BARBOSA, L.C.A.; AZEVEDO, A.A.; CASALI, V.W.D.; NASCIMENTO, E.A. Produção e composição do óleo essencial de alfavaquinha (Ocimum selloi Benth.) em resposta a dois níveis de radiação solar. Revista Brasileira de Plantas Medicinais, v. 6, n. 1, p.8-14, 2003.

GONZÁLEZ-ZÚÑIGA, J.A.; GONZÁLEZ-SÁNCHEZ, H.M.; GONZÁLEZ-PALOMARES, S.; ROSALESREYES, T.; ANDRADE-GONZÁLEZ. Microextracción en fase sólida de compuestos volátiles en albahaca (Ocimum basilicum L.). Revista Acta Universitaria. v.21, n.1, p.17-22, 2011.

HANIF, M.A.; AL-MASKARI, M.Y.; AL-MASKARI, A.; AL-SHUKAILI, A.; AL-MASKARI, A.Y.; AL-SABAHI, J.N. Essential oil composition, antimicrobial and antioxidant activities of unexplored Omani basil. Journal of Medicinal Plants Research, v.5, n.5, p.751-757, 2011.

LOAYZA, I.; ABUJDER, D.; ARANDAS, R.; JAKUPOVIC, J.; COLLIN, G.; DESLAURIERS, H.; JEAN, F.I. Essential oils of Baccharis salicifolia, B. latifolia and $B$. dracunculifolia. Phytochemistry, v.38, n.2, p.381-389, 1995.

LUZ, J.M.Q; MORAIS, T.P.S; BLANK, A.F.; SODRÉ, A.C.B.; OLIVEIRA, G.S. Teor, rendimento e composição química do óleo essencial de manjericão sob doses de cama de frango. Horticultura Brasileira, v.27, n.3, p.349-353, 2009.

KLEIN, T.1; LONGHINI, R.; BRUSCHI, M.L.; MELLO, J.C.P. Fitoterápicos: um mercado promissor. Revista de Ciências Farmacêuticas Básica e Aplicada.v.30, n.3, p.241-248, 2009.

MARTINS, F.T.; SANTOS, M.H.; POLO, M.; BARBOSA, L.C.A. Variação química do óleo essencial de Hyptis suaveolens (L.) Poit., sob condições de cultivo. Química Nova, v.29, n.6, p.1203-1209, 2006.

MARTINS, A.G. L.A.; NASCIMENTO, A.R.; FILHO, J.E.M.; FILHO, N.E.M.; SOUZA, A.G.; ARAGÃO, N.E.; SILVA, D.S.V. Atividade antibacteriana do óleo essencial do manjericão frente a sorogrupos de Escherichia coli enteropatogênica isolados de alfaces. Ciência Rural, v.40, n.8, p.1791-1796, 2010.

MATERN, U.; GRIMMIG, B. Natural phenols as stress metabolites. Acta Horticulturae, , v.58, n.381, p.448462, 1994.

NASCIMENTO, J.C.; BARBOSA, L.C.A.; PAULA, V.F.; DAVID, J.M.; FONTANA, R.; SILVA, L.A.M.; FRANÇA, R.S. Chemical composition and antimicrobial activity 
of essential oils of Ocimum canum Sims. and Ocimum selloi Benth. Anais da Academia Brasileira de Ciências, v.83, n.3, p.787-799, 2011.

OTTAI, M.E.S.; SAYEDA S.A.; EL DIN, M.M. Genetic Variability Among Some Quantitative Characters, Insecticidal Activity and Essential Oil Composition of Two Egyptian and French Sweet Basil Varieties. Australian Journal of Basic and Applied Sciences, v.6, n.3, p.185-192, 2012.

PIRES, M.J.P.; GRIPP, A. Conservação de recursos genéticos de plantas medicinais em banco ativo de germoplasma. Acta Amazônica, v.18, n.1/2, p.61-73, 1988.

RIBEIRO JÚNIOR, J.I.; MELO, A.L.P. Guia prático para utilização do SAEG. Viçosa: Editora UFV, 2009. 287p.

ROSADO, L.D.S.; PINTO, J.E.B.P.; BOTREL, P.P.; BERTOLUCCI, S.K.V.; NICULAU, E.S.; ALVES, P.B. Influência do processamento da folha e tipo de secagem no teor e composição química do óleo essencial de manjericão cv. Maria Bonita. Ciência e Agrotecnologia, v.35, n.2, p.291-296, 2011.

SAJJADI, S.E. Analysis of the essential oils of two cultivated basil (Ocimum basilicum L.) from Iran. Daru, v.14, n.3, p.128-130, 2006.

SILVA, F.; SANTOS, R.H.S.; ANDRADE, N.J.; BARBOSA, L.C.A.; CASALI, V.W.D.; LIMA, R.R.; PASSARINHO, R.V.M. Basil conservation affected by cropping season, harvest time and storage period. Pesquisa Agropecuária Brasileira, v.40, n.4, p.323-328, 2005. SILVA, C.J.; BARBOSA, L.C.A.; DEMUNER, A.J.; PINHEIRO, A.L.; DIAS, I.; ANDRADE, N.J. Chemical composition and antibacterial activities from the essential oils of Myrtaceae species planted in Brazil. Química Nova, v.33, n.1, p.104-108, 2010.

VELOSO, R.A.; CASTRO, H.G.; CARDOSO, D.P.; SANTOS, G.R.; BARBOSA, L.C.A.; SILVA, K.P. Composição e fungitoxicidade do óleo essencial de capim citronela em função da adubação orgânica. Pesquisa Agropecuária Brasileira, v.47, n.12, p.1707-1713, 2012.

VIÑA, A.; MURILLO, E. Essential Oil Composition from Twelve Varieties of Basil (Ocimum spp) Grown in Colombia. Journal of the Brazilian Chemical Society, v.14, n.5, p.744-749, 2003.

Rev. Bras. PI. Med., Campinas, v.16, n.2, supl. I, p.364-371, 2014. 